\title{
A Framework for Assessing the Economic Value of Pharmacovigilance in Low- and Middle-Income Countries
}

\author{
Joseph B. Babigumira • Andy Stergachis • \\ Hye Lyn Choi · Alexander Dodoo • \\ Jude Nwokike $\cdot$ Louis P. Garrison Jr.
}

Published online: 19 February 2014

(C) Springer International Publishing Switzerland 2014

\begin{abstract}
Pharmacovigilance (PV) programs are an essential component of national healthcare systems. Wellfunctioning PV programs can improve population health by identifying and reducing medicines-related problems (MRPs). Many low- and middle-income countries lack functional PV systems, but this deficiency has not been described in terms of the potential economic value of strengthening PV systems. The assessment of economic value for PV can support rational decision making at the country level. We propose a framework for assessing the economic value of PV. We divide national PV systems into four levels: (1) no PV, (2) basic PV, (3) semi-functional PV,
\end{abstract}

J. B. Babigumira $(\bowtie) \cdot$ A. Stergachis · L. P. Garrison Jr. Global Medicines Program, Department of Global Health, University of Washington, Harris Hydraulics Building, Room 319, 1705 NE Pacific St., Box 357965, Seattle, WA 98195-7965, USA

e-mail: babijo@uw.edu

J. B. Babigumira · A. Stergachis · L. P. Garrison Jr. Pharmaceutical Outcomes Research and Policy Program, Department of Pharmacy, University of Washington, Seattle, WA, USA

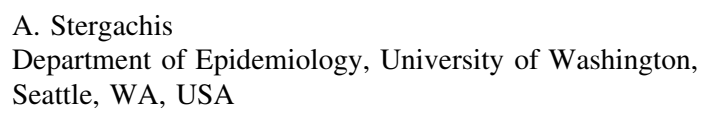

H. L. Choi

Geneva, Switzerland

A. Dodoo

World Health Organization Collaborating Centre

for Advocacy and Training in Pharmacovigilance, Accra, Ghana

J. Nwokike

Systems for Improved Access to Pharmaceuticals and Services (SIAPS) Program, Management Sciences for Health, Arlington, VA, USA and (4) functional PV. These categories represent increasing levels of investment in PV capacity at the national or health facility level for all available medicines, including vaccines. The proposed framework can be used to estimate the costs of PV (including the value of investments to increase PV capacity and the costs of managing MRPs) and outcomes associated with PV (including improvements in morbidity, mortality, and quality of life as a result of the reduction in MRPs). The quantitative approach proposed for assessing costs and benefits uses a decision-analytic modeling framework that would estimate the value of the consequences of MRPs adjusted for their probability of occurrence. This allows the quantification of value using monetary outcomes (cost-benefit analysis), natural units (cost-effectiveness analysis), or mortality adjusted for quality of life or disability (cost-utility analysis). Evidence generated using this framework could assist policy makers, program managers, and donors in evaluating investments that aim to increase the capacity and efficiency of national and facility-level PV programs in low- and middle-income countries.

\section{Key points}

Pharmacovigilance is a key but underfunded component of national healthcare programs in lowand middle-income countries.

A framework is proposed for assessing the economic value of pharmacovigilance programs.

Evidence generated using this framework could assist stakeholders in evaluating investments to increase the capacity and efficiency of pharmacovigilance and contribute to improving population health. 


\section{Background}

Pharmacovigilance (PV) is the science and activities relating to the detection, assessment, understanding, and prevention of adverse events (AEs) or other medicinesrelated problems (MRPs) [1]. The conduct of PV includes spontaneous reporting of suspected AEs, focused active surveillance studies, the use of confirmatory pharmacoepidemiologic studies, and risk mitigation and risk communication strategies [1]. PV is an essential component of public health programs but countries have different levels of PV activity [2]. The World Health Organization (WHO) recommends that evaluation and assessment be built into PV monitoring systems with specific performance criteria such as: quality of reporting, specialization of reporting person, reporting of novel adverse drug reactions (ADRs), promptness of reporting, effectiveness of reporting, health outcomes, and costs [2]. The WHO Program for International Drug Monitoring is also developing a list of performance indicators including structural, process, and outcome (impact) indicators [3]. The impact indicators refer to "efficient and safe use of medicines" without explicit mention of economic value [3]. A proposed outline for good PV practice proposed by a group of EU PV experts identifies the measurement of expected and obtained effects as a challenge to good PV practice [4].

It is important to demonstrate that $\mathrm{PV}$ is an essential public health function with sound processes and the ability to improve health outcomes. It is also essential to demonstrate that the level or scale of PV is cost effective [3]. However, even in high-income countries where PV is well developed, systematic assessments of the economic value of PV are not routine or even generally available. However, studies have demonstrated the substantial mortality burden [5] and economic loss associated with adverse drug events [6-8]. A meta-analysis of 39 prospective studies in the US found that ADRs were a leading cause of death [5]. A comprehensive review found that AEs were associated with between 1 and 4 additional days of hospitalization and \$US2,300-5,600 in additional hospital costs [6]. In the US, the treatment of MRPs was estimated to cost \$US177 billion in 2000 [6]. In the UK, the projected annual cost of admission related to ADRs to the National Health System was $£ 847$ million in 2004 [8]. Studies have demonstrated the economic impact of different individual treatments in terms of their cost per $\mathrm{AE}$ prevented [6], but rigorous assessments of the cost or cost effectiveness of national PV systems have not been reported; published reports focus on the mortality and hospitalization attributable to AEs. Yet it is generally recognized that PV can protect the public's health by identifying the risks of and the risk factors for drug AEs in a timely manner and using information for risk mitigation. Information collected through PV allows for the assessment of the risks and benefits of a medicinal product throughout its life cycle [9].

Although PV is considered to be an essential public health function, PV programs are not immune to fiscal constraints. An analysis of 55 low- and middle-income countries (LMICs) found considerable variation in the infrastructure, resources, and practices of $\mathrm{PV}$ programs [10]. According to Roll Back Malaria, a national PV program for anti-malarial agents is estimated to cost from $\$ 150,000$ to $\$ 250,000$ for start-up with recurrent costs of around $\$ 50,000$ per year. However, many LMICs lack some or all of the WHO's basic elements of a PV system [11]. Most countries in sub-Saharan Africa have some PV activities, but the majority have not organized these activities into comprehensive national PV systems [12].

A country's lack of a functional PV system leads to greater costs in terms of the resources used to manage and prevent MRPs as well as worse health outcomes in terms of medicines-related morbidity and mortality and medicinesrelated quality-of life (QoL) reductions and disability. Quantifying these impacts in terms of the opportunity cost of the resources used and the adverse health impacts is important in assessing the potential value of starting or strengthening national PV centers. In LMICs, limited healthcare budgets mean that new lines of expenditure, such as would be required to start or strengthen national PV systems, would likely be scrutinized. We propose a framework for the assessment of the economic value of PV programs. This framework is intended to enable policy makers, healthcare planners, and other stakeholders to assess the value of potential or planned investments in PV, or to support the fiscal case for an appropriate level of PV funding.

\section{Levels of Investment in National Pharmacovigilance Systems}

Countries can undertake different levels of investment in $\mathrm{PV}$ on a continuum from having no system at all to having a fully functional system. The minimum requirements for a functional PV system at the national level, as defined by the WHO, are a PV center with designated staff, a spontaneous reporting system, a database or system for collating and managing adverse drug reaction (ADR) reports, a PV advisory committee, and a communications strategy [11]. These requirements formed the basis of a recently published classification of levels of PV in sub-Saharan Africa [12], in which national PV systems were ranked according to their performance in five areas: (1) policy, law, and regulation; (2) system, structure, and stakeholder coordination; 
(3) signal generation and data management; (4) risk assessment and evaluation; and (5) risk management and communication [4]. Countries were classified into four levels. In level 1 (no PV), there are no legal or structural frameworks, no coordinated surveillance activities, and PV activities are not coordinated nationally. In level 2 (basic PV), policy and legal frameworks exist, institutions, guidelines, and procedures exist, and stakeholder roles are recognized albeit poorly coordinated. Additionally, the AE reporting system does not cover all sources of MRPs, signal generation and risk evaluation are poor, and the system lacks active signal evaluation and risk management. In level 3 (semi-functional PV), structural and organizational frameworks exist to collect and collate safety data and evaluate risks and benefits by passive and active surveillance. However, the countries lack risk management, risk prevention, and risk communication capacity. In level 4 (functional PV), a PV structure exists that permits passive and active surveillance, risk evaluation, risk communication, and regulatory action.

The proposed framework aims to answer the question for a given country or health facility for all medicines and vaccines and all indications: what would be the economic value of investing new resources to move from a less to any one of the more sophisticated levels of PV? We emphasize new resources because we do not propose to estimate the opportunity cost of moving resources from other health services. Countries at lower levels of PV may not aim to directly achieve level 4 status but rather improve progressively while estimating the economic value for the benefit of stakeholders.

Any investment in PV and the assessment of the potential economic value should consider the substantial diversity in country characteristics. Some countries are larger than others in size and population, and this has an impact on the nature and amount of potential investments in PV. Because of this diversity, the proposed framework should be customized at the country level taking into consideration a country's relevant unique characteristics, such as population and geography.

\section{Proposed Analytic Framework}

The proposed framework for assessing the economic value of PV is shown in Fig. 1. The framework allows the estimation of the costs and outcomes of investing in PV systems. On the cost side, it includes the costs of PV activity and MRPs at different levels of PV. On the outcomes side, it includes different valuations of the health outcomes and benefits of increasing PV activity. Thus, economic value is defined in terms of cost savings plus the value of the reductions in mortality and morbidity.

\subsection{Methods of Assessing Economic Value}

The economic value of healthcare programs can be assessed by quantifying and comparing both their costs and benefits in formal evaluations using different methods. The comparison of the costs is uniform across methods but the quantification of benefits varies from method to method.

In a cost-benefit analysis (CBA), the net costs of a healthcare intervention (such as movement from a lower to a higher level of PV) are compared with the benefits of the intervention expressed in monetary units. The metric of economic value is the net benefit, a measure of the benefit minus the net cost. A positive net benefit is a general signal to policy makers to fund the intervention. The benefit-tocost ratio can also be estimated as the metric for value; the higher the ratio the better the investment. A benefit-to-cost ratio of, say, 4 implies that every dollar invested yields 4 dollars in return. For example, the benefit-to-cost ratio for measles and rubella vaccination in the general population has been estimated to be as high as 14 [13]. Despite being arguably the most complete method of economic evaluation, CBA is disadvantageous because it is difficult to monetize clinical and QoL outcomes [14]. Additionally, the main method for the said monetary conversion-willingness to pay-gives greater weight to the preferences of wealthy people, making it ethically unpalatable to many [14].

Other methods of assessing the economic value that include health outcomes without monetization exist and can be used. In this case, the metric of assessing value is a ratio of the incremental cost to the incremental benefit or health outcome-the incremental cost-effectiveness ratio (ICER). In cost-effectiveness analyses (CEAs), the outcomes are presented in natural units such as number of AEs averted. Economic value in this case would be expressed as cost per AE averted. Cost-utility analyses (CUAs) combine length of life and QoL in the outcome measure. The combined metric is presented as the quality-adjusted lifeyear (QALY) or disability-adjusted-life-year (DALY). Economic value is expressed as an ICER (cost per QALY gained or cost per DALY averted).

\subsection{Estimating the Costs at Different Levels of Pharmacovigilance}

\subsubsection{Estimating the Costs of PV-Related Activities}

The investment needed at each of the different PV levels can be estimated by applying unit costs to the different types and volumes of human and physical resources required. The resources needed increase as PV systems become more sophisticated but are also tied to other factors 
Fig. 1 Framework for assessing the economic value of pharmacovigilance in low- and middle-income countries. $P V$ pharmacovigilance, $M R P S$ medicine-related problems, $O P$ out-patient

\section{COSTS}

Increasing: PV Activity e.g. Space, Equipment, Personnel, Materials

Decreasing: MRPs e.g. OP visits, Hospitalizations, Regimen Switches, Lost Work

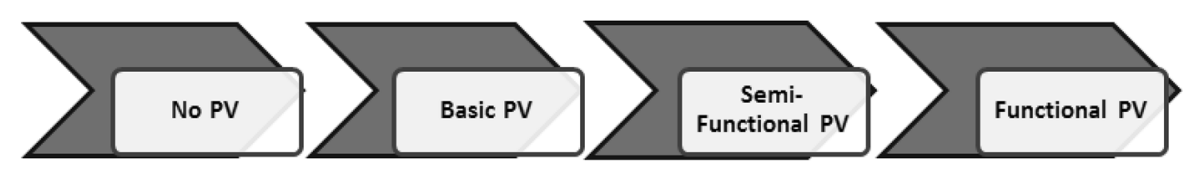

OUTCOMES

Increasing: quality-of-life and productivity

Decreasing: morbidity and mortality
Table 1 Examples of resources required for implementing pharmacovigilance at the national level

\begin{tabular}{l}
\hline Capital resources \\
Equipment \\
Office furniture \\
IT equipment \\
Vehicles \\
Books \\
Recurrent (monthly) resources \\
Office space ( ${ }^{2}$ ) \\
Personnel \\
Physicians \\
Pharmacists \\
Nurses \\
Clinical pharmacologist \\
Epidemiologist \\
Driver \\
Support staff \\
Materials and supplies \\
Utilities \\
Travel \\
Mass media \\
Meetings \\
Serial publications \\
Web hosting \\
Database subscriptions
\end{tabular}

such as population, geography, and road and information technology infrastructure. For example, as the level of PV increases, expenditure on physician wages increases. The costs of PV can be divided into capital and recurrent costs/ resources. Examples of these are shown in Table 1. The sum of the product of all resources and their respective unit costs can be computed and represents the value of the investment required to move from one level of $\mathrm{PV}$ to another in a given country or health facility.

\subsubsection{Estimating the Costs of MRPs}

The costs of managing different drug AEs and other MRPs include: (1) cost of out-patient (OP) visits, (2) cost of hospitalization, and (3) cost of MRP-related regimen switches including new drugs and consultations. Costs of OP visits and hospitalization for MRPs include direct medical costs (such as health workers time, other medications or antidotes, and laboratory tests), direct nonmedical costs (such as patient transportation and upkeep), and indirect costs (which include the opportunity cost of lost productivity during MRP-related illness and convalescence).

\subsection{Estimating Outcomes}

\subsubsection{Monetizing the Benefits of Pharmacovigilance for Cost-Benefit Analysis}

PV involves the appraisal and prevention of AEs and other MRPs. The benefits of PV from a cost-benefit standpoint are a valuation of improved health outcomes associated with the reduced incidence of AEs and MRPs including reduced morbidity and mortality and improved QoL. In addition to these traditional health outcomes, non-traditional patient outcomes such as risk protection (greater peace of mind from knowing that appropriate PV system is in place), option values (the benefits of having a PV program available in the event that it is needed), and altruistic values (the benefits of seeing others benefit from the availability of a PV program) can also be included in the valuation. The health outcomes benefits may be valued using the human capital approach (in which the value of an individual's contribution is tied to their wage) and willingness to pay (in which the value is based on how much an individual is willing to accept for an increased risk of the outcome or pay for access to a service to manage the outcome) [14]. Bouvy et al. [15] applied willingness-to-pay 
methods to monetize the health outcomes associated with regulatory action to reduce the risk of pure red cell aplasia associated with the use of epoetin alpha. They found that Dutch dialysis patients were willing to pay significantly higher $(€ 47)$ than the Dutch general population (€24) [15]. Estimates generated in this manner can be used in a CBA of national level PV programs.

\subsubsection{Estimating Outcomes for Cost-Effectiveness Analysis and Cost-Utility Analysis}

A variety of health outcomes can be estimated for CEA including number of AEs, number of OP visits and hospitalizations, number of regimen switches, and mortality averted (measured in life-years saved). For example, a study was conducted to compare non-steroidal anti-inflammatory drugs (NSAIDS) in the management of rheumatoid arthritis and osteoarthritis with cost per life-year gained as an outcome [16]. Data from several studies [16, 17] were used to compare different NSAIDS in a study in which the metric for economic value was the cost per AE avoided [6]. These metrics may be used in assessing the health outcomes associated with increasing PV activity at the country level. To perform a CUA, the impact of MRPs on QoL or disability would need to be estimated across a number of indications. This requires the performance of patientreported outcomes studies and potentially, the use of publically available data sources. In the future, data from social media may become available for use in studies [18-20]. If the measure of QoL includes a valuation of the impact of work loss associated with MRPs, the costs of lost work should not be included in the numerator of the cost-utility ratio, i.e., the indirect costs of MRPs should be excluded.

\subsection{Decision-Analytic Framework}

The estimation of the costs and health outcomes needed to measure economic value requires a modeling framework to quantify the sequential occurrence of events as well as their probability. For instance, the cost of MRPs is a function of the unit costs of MRPs and the probability of their occurrence. A modeling framework can combine estimates of the reduction in the probability of relevant MRPs due to increasing PV activity and their costs.

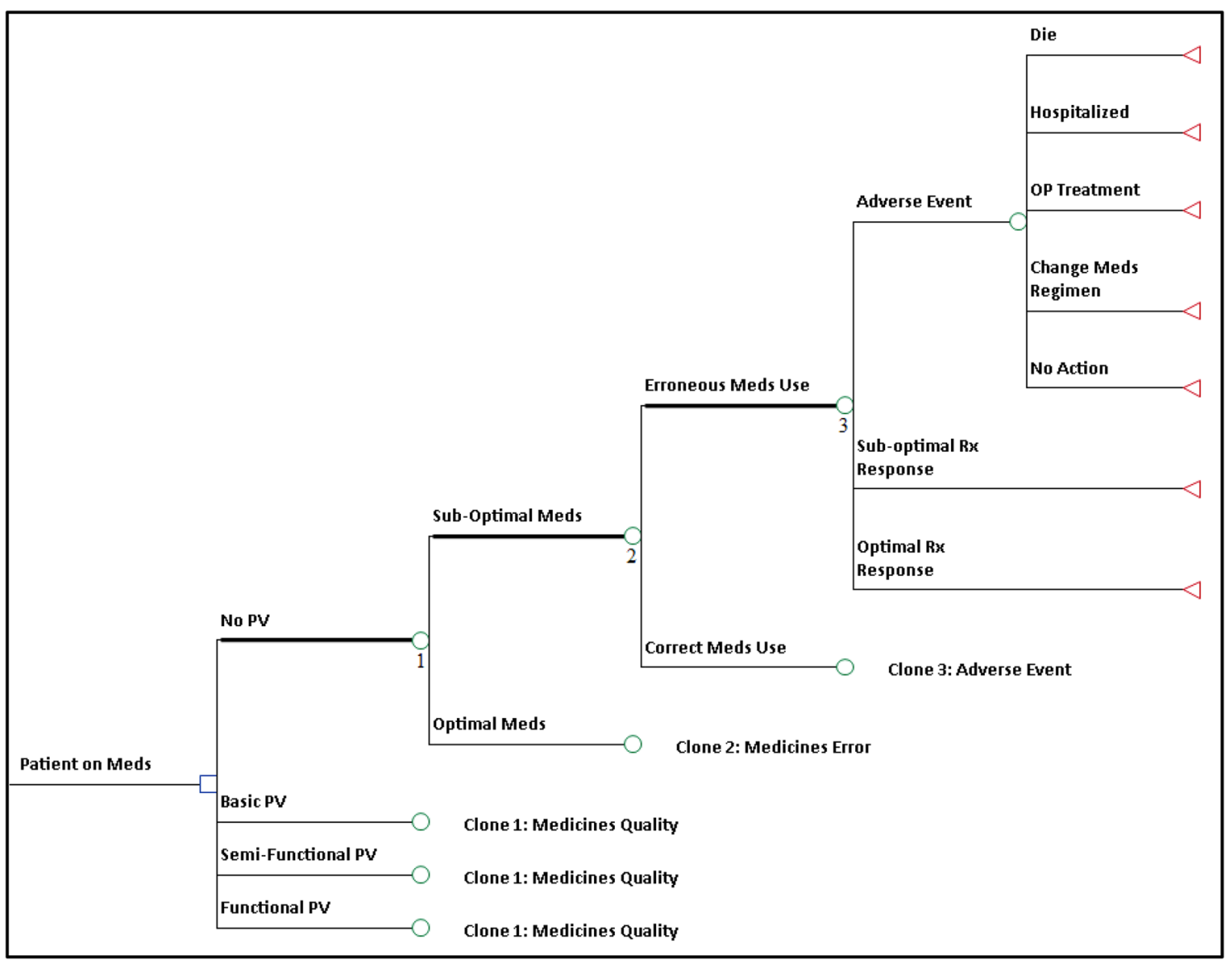

Fig. 2 Proposed decision-analytic model showing possible medicines-related problems and different levels of investment in pharmacovigilance. Meds medicines, $P V$ pharmacovigilance, $R x$ treatment, $O P$ out-patient 
A decision-analytic modeling framework such as the one shown in Fig. 2 can be used to organize and estimate the costs and outcomes of PV. Decision-analytic modeling uses statistical tools including decision trees, probability theory, multivariate analysis, and probabilistic techniques to assess the impact of different decisions, such as policy decisions under different conditions including conditions of uncertainty. Decision analysis is the most common model for conducting CEA in pharmacoeconomics and is increasingly used for healthcare policy decisions in LMICs. It has been used to estimate the national level costs of drug AEs in the out-patient setting in Germany [21].

The model is used to organize the MRPs that can occur and the consequences of different levels of investment in $\mathrm{PV}$. The reference case in the model is a patient on medication for a given indication. This patient may live in an area in which one of the four levels of PV is in effect as shown at the decision node (square in Fig. 2).

Basic PV is the minimum level of investment to reduce MRPs. Semi-functional PV implies an improvement on basic PV and functional PV implies an improvement on semi-functional PV. While comparators in traditional decision-analytic models should be mutually exclusive, these PV levels appear not to be: they are incremental, one building on the other. In this framework, we consider them to be mutually exclusive in the sense that one area would have only one of the levels of PV operating at a given time.

Given a patient's residence in an area with a certain level of PV, there will be a set of probabilities of different MRPs at chance nodes (circles in Fig. 2). The medicines that the patient receives may be optimal or sub-optimal. Sub-optimal medicines include fake, substandard, expired, or otherwise defective medicines. In the next phase of the model, the patient may use the medicines correctly or incorrectly. Erroneous medicine use may be a result of irrational prescribing, poor dispensing, or poor use of medicines by patients, e.g., non-adherence. According to the use of the medicines, erroneous or correct, the patient may or may not exhibit adverse drug events, exhibit a suboptimal treatment response, or exhibit an optimal treatment response. If the patient exhibits adverse drug events, there are at least five possible outcomes: (1) MRP-related death, (2) MRP-related hospitalization, (3) MRP-related outpatient visit, (4) a change of medicine regimen, and (5) no action, i.e., the $\mathrm{AE}$ is non-fatal and the patient faces its consequences without intervention. At the end of the model, the end nodes (triangles in Fig. 2) represent payoffs or outcomes. For a given model scenario, payoffs are assigned depending on the outcome. For instance, for a mortality outcome, the "die" branch is assigned a value of 1 while all other branches are assigned a value of 0 . For a survival outcome, the "die" branch would be assigned a value of 0 and all other branches a value of 1 . The model can be set up to calculate the different payoffs and generate the data required to perform CBA, CEA, and CUA.

\subsubsection{Model Inputs}

The inputs into the modeling framework include: probabilities of different events (e.g., sub-optimal medicines use, erroneous medicines use, AEs, out-patient visits, hospitalizations, regimen changes) and the costs of different MRPs. There are two possible alternative methods to estimate changes in probabilities: (1) estimate directly the reductions in probability of AEs if more resources are invested in $\mathrm{PV}$, or (2) estimate the probabilities of AEs in countries where a given level of PV is being implemented and take the difference.

\section{Discussion}

We herein propose a quantitative framework for the estimation of the potential economic value of PV programs in LMICs. This framework estimates costs (of investments in increased PV activity and management of MRPs) and outcomes as monetary values (CBA), averted AEs, or lifeyears (CEA), and quality- or disability-adjusted life expectancy (CUA). The estimates required to perform CBA, CEA, and CUA can be obtained using a decisionanalytic model with probabilities estimated directly or as a percentage reduction in probability of MRPs as a result of increasing PV activity. The choice of value assessment should be guided by availability of data and technical expertise but should be transparent and clear.

This framework can guide the development of useful analytic tools with user-defined inputs that can be used to make the case for an appropriate, efficient level of PV funding. For a given country or health facility, the challenge would be to generate data to implement the framework. As described above, four key inputs need to be estimated: (1) the monetary value of the investment, (2) the costs of managing different MRPs, (3) the probability of different events including reductions in MRPs as a result of increased PV activity, (4) the impact of MRPs on QoL or disability. Most of these data are difficult and costly to collect in most settings. Unit cost data at the country level are likely to be less challenging to collect or estimate than data on probabilities.

A variety of types of studies are needed to generate estimates, including systematic reviews of the literature, database analyses, and Delphi surveys with panels of experts. A challenge is the complexity associated with estimating reductions in MRPs as a result of increasing PV activity. This is because PV investments are made at the national level for patients with multiple diseases yet events 
are indication and medicine specific. Further, some estimates exist in the literature of the potential reduction in specific events associated with certain medications (e.g., antimalarial agents) for a given indication [22, 23], but to estimate events at the national level requires aggregating and integrating data from multiple sources and databases. Even with aggregated data, estimates across indications and medicines for a given country will likely have a great deal of uncertainty surrounding them. Given the difficulty and cost of collecting data as well as the potential uncertainty, the implementation of this framework would have to (1) use univariate, threshold, and multivariate sensitivity analyses and (2) examine the impact of key estimates (such as percent reduction in AEs) on the cost-benefit ratio, the ICER, or the incremental cost-utility ratio. Therefore, even when good-quality data are difficult or costly to obtain, studies of the potential economic value of PV should be performed and might be valuable to policy makers.

In selecting the optimal level of PV in a given LMIC, it will be important to recognize the "public good" nature of information, in that any given country can benefit from the experiences in other countries. For example, if a specific medicine is generating excessive ADRs in another country, authorities may be able to act based on that information. Thus, in a region, what is "efficient pharmacovigilance" will depend on whether there is an information-sharing network. For instance, countries that are full members of the WHO Programme for International Drug Monitoring may use access to Vigibase - the WHO database of AEsto their benefit.

\section{Recommendations and Next Steps}

This framework should be tested using real-world data to test the assumptions as well as structural validity. In further developing this framework into a user-friendly tool, we anticipate some challenges that will require additional research. The measurement of MRPs will need to be studied in greater detail. Our proposed approach using decision-analytic methods assumes the individual patient as the level of analysis. This might not be optimal as the problem might be better formulated as a "multi-product cost function" as might be done for a hospital serving a given population, where the outputs are defined by different procedures or types of visits. It may also be better to perform analyses based on public health programs such as malaria, HIV/AIDS, or tuberculosis programs with benefits assigned to the program as a whole. In this case, other analytic methods such as discrete event simulation (DES) might be considered given multiple levels of PV activity, multiple indications, and multiple prescriptions per individuals. Additionally, the proposed decision-analytic framework is limited in its consideration of time: it considers costs and outcomes only over short time periods. Given that PV systems may take a long time to develop methods such as Markov models and DES may be more appropriate.

Another anticipated challenge is the estimation and incorporation into the analytic framework of economies of scale and economies of scope as PV capacity is increased, particularly for larger countries. Ideally, the complete framework could be implemented as a software-based userfriendly and interactive tool that is customizable for use at the national level to estimate the economic value of PV and, at a later stage, a software application for personal computers, tablets, and smartphones that would put the ability to measure economic value in the hands of a wide variety of stakeholders in medicine safety.

\section{Conclusions}

PV systems have the potential to improve health outcomes and to reduce healthcare expenditures related to AEs. However, because of severe resource constraints in LMICs, policy makers and donors must scrutinize investments to obtain value for money. This framework is designed to support decision making about whether greater funding for PV would be efficient and desirable. A fully developed tool to assess economic value could assist policy makers and donors in evaluating investments required to increase the capacity of national programs to improve the use, safety, quality, cost effectiveness, and affordability of medicines in LMICs.

Acknowledgments Hye Lyn Choi and Jude Nwokike are former employees of Management Sciences for Health (MSH), which implemented the USAID-funded Strengthening Pharmaceutical Systems (SPS) Program. Funding for this work was obtained from the US Agency for International Development (USAID), under the terms of cooperative agreement number GHN-A-00-07-00002-00. The contents are the responsibility of the authors and do not necessarily reflect the views of USAID or the United States Government. Joseph B. Babigumira, Andy Stergachis, Hye Lyn Choi, Alexander Dodoo, Jude Nwokike, and Louis P. Garrison, Jr. have no other conflicts of interest that are directly relevant to the content of this article.

\section{References}

1. The Importance of Pharmacovigilance: World Health Organization. Geneva; 2002.

2. The safety of medicines in public health programmes: pharmacovigilance an essential tool. World Health Organization; 2006. http://www.who.int/medicines/areas/quality_safety/safety_efficacy/ Pharmacovigilance_B.pdf.

3. Edwards IR, Isah A. Pharmacovigilance and the null hypothesis: do we do much for public health? Drug Saf. 2011;34(2):93-6. 
4. Pietrek M, Coulson R, Czarnecki A. Good pharmacovigilance practice: the way forward? Drug Inf J. 2009;43(5):623-32.

5. Lazarou J, Pomeranz BH, Corey PN. Incidence of adverse drug reactions in hospitalized patients: a meta-analysis of prospective studies. JAMA. 1998;279(15):1200-5.

6. Rodriguez-Monguio R, Otero MJ, Rovira J. Assessing the economic impact of adverse drug effects. Pharmacoeconomics. 2003;21(9):623-50.

7. Chiatti C, Bustacchini S, Furneri G, Mantovani L, Cristiani M, Misuraca $\mathrm{C}$, et al. The economic burden of inappropriate drug prescribing, lack of adherence and compliance, adverse drug events in older people: a systematic review. Drug Saf. 1007;35(Suppl 1): 73-87.

8. Pirmohamed M, James S, Meakin S, Green C, Scott AK, Walley TJ, et al. Adverse drug reactions as cause of admission to hospital: prospective analysis of 18820 patients. BMJ. 2004;329(7456):15-9.

9. Strengthening Pharmaceutical Systems (SPS). Supporting pharmacovigilance in developing countries. USAID, Arlington: Management Sciences for Health; 2009.

10. Olsen S, Pal S, Stergachis A, Couper M. An analysis of pharmacovigilance activities in 55 low- and middle income countries. Drug Saf. 2010;33:689-703.

11. Minimum requirements for a functional pharmacovigilance system. http://www.who.int/medicines/areas/quality_safety/safety_ efficacy/PV_Minimum_Requirements_2010_2.pdf.

12. Strengthening Pharmaceutical Systems (SPS) Program. Safety of Medicines in Sub-Saharan Africa: Assessment of Pharmacovigilance Systems and their Performance. Submitted to the US Agency for International Development by the Strengthening Pharmaceutical Systems (SPS) Program. Arlington: Management Sciences for Health; 2011.

13. Babigumira JB, Morgan I, Levin A. Health economics of rubella: a systematic review to assess the value of rubella vaccination. BMC Public Health. 2013;13(406):406.
14. Robinson R. Cost-benefit analysis. BMJ. 1993;307(6909):924-6.

15. Bouvy J, Weemers J, Schellekens H, Koopmanschap M. Willingness to pay for adverse drug event regulatory actions. Pharmacoeconomics. 2011;29(11):963-75.

16. McCabe CJ, Akehurst RL, Kirsch J, Whitfield M, Backhouse M, Woolf AD, et al. Choice of NSAID and management strategy in rheumatoid arthritis and osteoarthritis: the impact on costs and outcomes in the UK. Pharmacoeconomics. 1998;14(2):191-9.

17. Bentkover JD, Baker AM, Kaplan H. Nabumetone in elderly patients with osteoarthritis: economic benefits versus ibuprofen alone or ibuprofen plus misoprostol. Pharmacoeconomics. 1994;5(4):335-42.

18. Edwards IR, Lindquist M. Social media and networks in pharmacovigilance: boon or bane? Drug Saf. 2011;34(4):267-71.

19. Knezevic MZ, Bivolarevic IC, Peric TS, Jankovic SM. Using Facebook to increase spontaneous reporting of adverse drug reactions. Drug Saf. 2011;34(4):351-2.

20. Garrard EE. Social media and postmarketing drug safety. Monitor; 2011. http://www.drugsafetyalliance.com/publications/ 12419-07_Garrard.pdf.

21. Stark R, John J, Leidl R. Health care use and costs of adverse drug events emerging from outpatient treatment in Germany: a modelling approach. BMC Health Serv Res. 2011;11(1):9.

22. Mehta U, Durrheim D, Mabuza A, Blumberg L, Allen E, Barnes KI. Malaria pharmacovigilance in Africa: lessons from a pilot project in Mpumalanga Province, South Africa. Drug Saf. 2007;30(10):899-910.

23. Dube NM, Summers R, Tint KS, Mayayise G. A pharmacovigilance study of adults on highly active antiretroviral therapy, South Africa: 2007-2011. Pan Afr Med J. 2012;11(39):39. 